\title{
The CGM Range Analysis based on 5-minute measurement intervals from the CGM sensor glucose data using GH-Method: Math-physical medicine
}

Gerald C. Hsu

EclaireMD Foundation, USA

\author{
"Correspondence author \\ Gerald C Hsu \\ EclaireMD Foundation \\ USA
}

Submitted : 2 June 2020 ; Published : 18 June 2020

\section{Introduction}

The author utilized the American Diabetes Association (ADA) 2020 Guidelines for time-in-range (TIR), time-above-range (TAR), and time-below-range (TBR) to analyze his collected CGM sensor glucose data for every 5-minute measurement interval (5-min). He also compares two different results based on the ADA definition and a customized definition (GH model). The author has termed this as the "CGM Range Analysis".

\section{Methods}

A continuous glucose monitor (CGM) device has been placed on his left upper arm to collect 54,720 glucose data over 724 days from 5/5/2018 - 4/28/2020, at a rate of 75.58 glucoses per day. These data were collected approximately every 15 minutes during the daytime and every hour during nighttime (15-min).

Furthermore, by using Bluetooth technology, on 2/19/2020, he installed an additional electronic device on top of the CGM sensor to collect and transmit the glucose data from the sensor directly to his developed iPhone APP at 5-minute intervals. For a period of 69 days $(2 / 19 / 2020$ - 4/28/2020), he has collected 17,871 glucose data at approximately 259 measurements per day.

Recently, the ADA published revised guidelines regarding CGM collected data (References 1 and 2). The new guidelines include the following three measurement terms:

1. TIR: time-in-range $70-180 \mathrm{mg} / \mathrm{dL}$ for "acceptable" diabetes glucose range.

2. TAR: time-above-range $>180 \mathrm{mg} / \mathrm{dL}$ for severe diabetes concerns.

3. TBR: time-below-range $<70 \mathrm{mg} / \mathrm{dL}$ for insulin shock warning.

A conversion table between glucose TIR and $\mathrm{HbA} 1 \mathrm{C} \%$ is shown in Table 1. After the ADA's announcement, many researchers have written papers about TIR (References 3, 4, and 5). The author also contributed several medical papers regarding the CGM Range Analysis (References 6, 7, and 8).

\begin{tabular}{|l|l|}
\hline Time-in-range & HbAlc $(\%)$ \\
\hline $0 \%$ & 12.1 \\
\hline $10 \%$ & 11.4 \\
\hline $20 \%$ & 10.6 \\
\hline $30 \%$ & 9.8 \\
\hline $40 \%$ & 9.0 \\
\hline $50 \%$ & 8.3 \\
\hline $60 \%$ & 7.5 \\
\hline $70 \%$ & 6.7 \\
\hline $80 \%$ & 5.9 \\
\hline $90 \%$ & 5.1 \\
\hline $100 \%$ & 4.3 \\
\hline
\end{tabular}

Table 1: ADA-based conversion table between TIR \% and $\mathrm{HbA} 1 \mathrm{C}$

He has observed his own diabetes conditions for 10 years and collected several hundred thousand data about his medical conditions. Therefore, he decided to define a customized TIR range between $70 \mathrm{mg} / \mathrm{dL}$ and $140 \mathrm{mg} / \mathrm{dL}$ (GH Model) in order to have a more stringent criteria for his own diabetes control, but still follows the ADA's general idea and concept.

\section{Results}

The 5-min dataset only covers 69 days with 259 measurement counts per day, while the 15-min dataset covers 724 days with $\sim 76$ measurements per day. Therefore, the author selected the same period of 69 days from 2/19/2020 through 4/28/2020 for a comparison study. 
Figure 1 shows a 5 -min comparison results of both TIR $\%$ and TIR average glucose in $\mathrm{mg} / \mathrm{dL}$ for the ADA Model (70-180mg/ dL) vs. customized GH Model (70-140mg/dL).

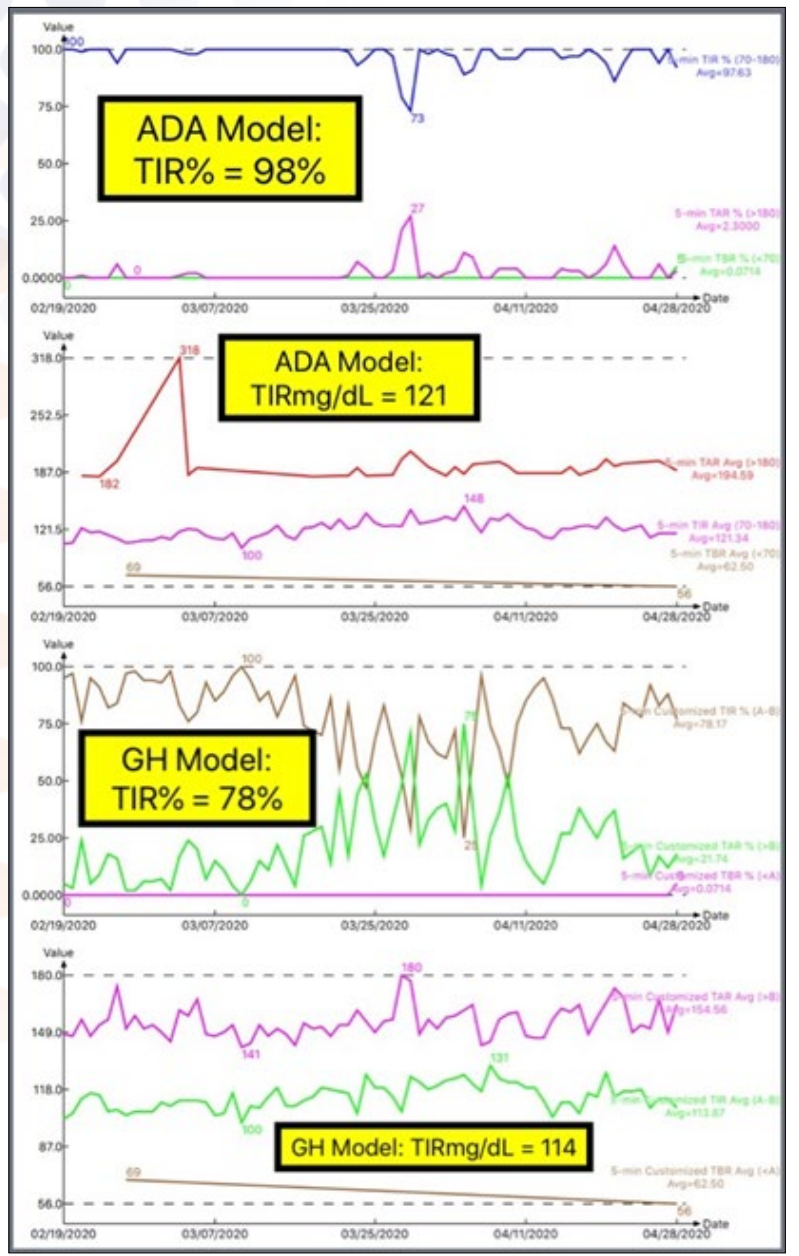

Figure 1: CGM Range Analysis of \% and average glucose for both ADA Model and Customized GH Model

From Figures 2, 3, and 4, a significant difference of TIR \% between the ADA Model (98\%) and GH Model (78\%) is observed, while a moderate difference of average glucose between the ADA Model $(121 \mathrm{mg} / \mathrm{dL})$ and GH Model (114 $\mathrm{mg} / \mathrm{dL}$ ) is detected. To date, he has not encountered the risk of insulin shock, both TBR are $0.1 \%$.

\begin{tabular}{|l|c|c|}
\hline ADA 5-min (2/19-4/28) & ADA $\%$ & GH \% \\
\hline TIR (70-180) & $98 \%$ & $78 \%$ \\
\hline TAR $(>180)$ & $2 \%$ & $22 \%$ \\
\hline TBR $(<70)$ & $0.1 \%$ & $0.1 \%$ \\
\hline Customized 5-min & ADA mg/dL & GH mg/dL \\
\hline TIR (70-140) & 121 & 114 \\
\hline TAR $(>140)$ & 195 & 155 \\
\hline TBR $(<70)$ & 63 & 63 \\
\hline
\end{tabular}

Figure 2: Table of Percentages and average glucose values of TIR, TAR, and TBR for both ADA Model and Customized GH Model

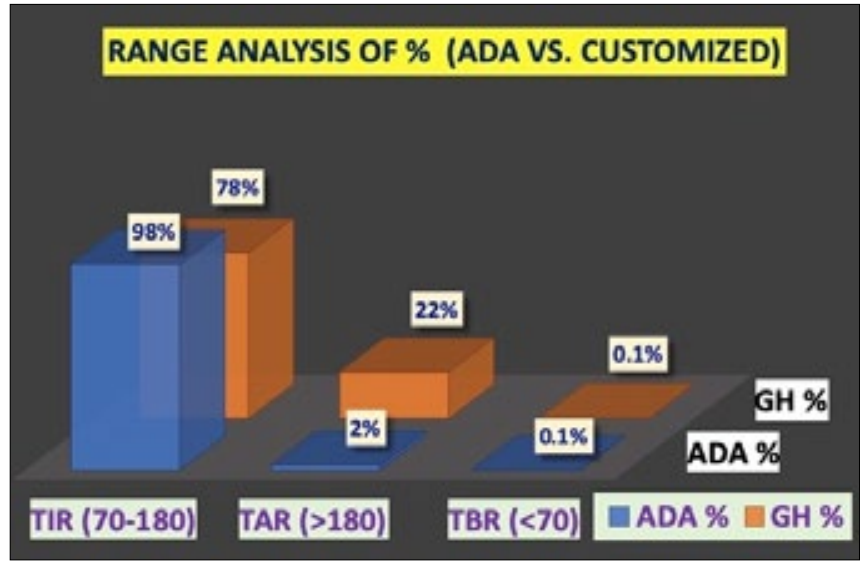

Figure 3: Bar chart of Percentages of TIR, TAR, and TBR for both ADA Model and Customized GH Model

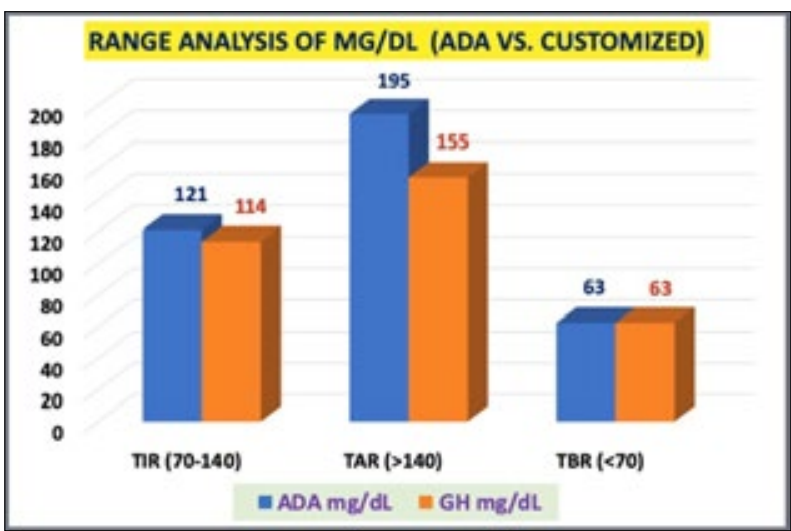

Figure 4: Bar chart of Average glucoses of TIR, TBR, and TAR for both ADA Model and Customized GH Model

However, according to the conversion table in Figure 1, the ADA Model's TIR of $98 \%$ would correspond to a value of $\mathrm{HbA1C}$ at $4.9 \%$ level, while the GH Model's TIR of $78 \%$ would correspond to a value of $\mathrm{HbA} 1 \mathrm{C}$ at $6.5 \%$ level. Thus far, the author has developed two mathematical models to predict his two sets of daily $\mathrm{HbA1C}$ values based on both finger-piercing measured glucoses (6.49\%) and CGM sensor collected glucoses $(6.52 \%)$. Therefore, he can safely state that his average $\mathrm{HbA1C}$ during this time period is around a $6.5 \%$ level (Figure 5). This further means that the customized GH Model's results (TIR 78\% and HbA1C 6.5\%) are a better match to his actual diabetes conditions.

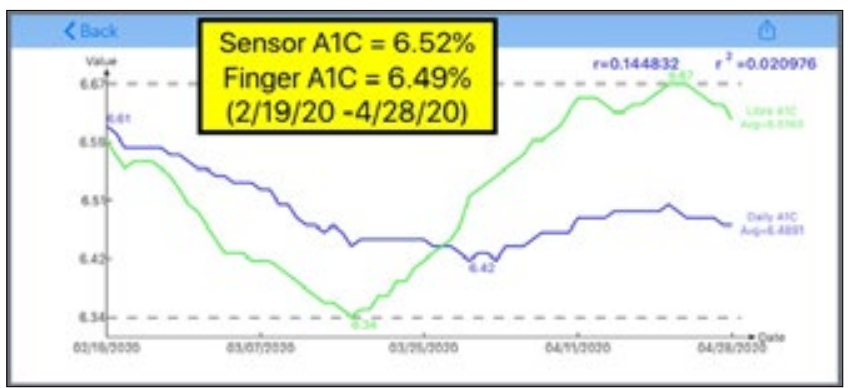

Figure 5: Line chart of Daily HbA1C values for both Finger glucoses and CGM sensor glucoses 
The main reason he collects his 5-min glucose data is that he can then utilized them to conduct a wave theory and frequency domain analysis. In addition, he can also apply the energy theory analysis to investigate the behavior of those higherfrequency with lower-amplitude glucose components and then investigate their impacts on our human internal organs (i.e. diabetes complications research).

This research paper demonstrates that the CGM glucose data provides an overall detailed comprehensive picture of a diabetes patient's glucose profile. However, after reviewing his own results of 17,871 CGM sensor collected data within 69 days, the author decided to define a tighter range of TIR between $70 \mathrm{mg} / \mathrm{dL}$ to $140 \mathrm{mg} / \mathrm{dL}$ (commonly accepted as the beginning level of diabetes), instead of using the ADA's 70 $\mathrm{mg} / \mathrm{dL}$ to $180 \mathrm{mg} / \mathrm{dL}$ (beginning of severe stage of diabetes). With this tighter customized TIR range, he could derive a TIR percentage which is closer to his lab-tested or mathematically predicted average $\mathrm{HbA} 1 \mathrm{C}$ value. By checking his past history of glucose conditions over the last 10-years, his customized definition seems to be a better fit with his present diabetes situation. This can be a conclusive observation from the ADA's recommendation based on a possible fact that most of their patients were under the influence of medications, while the author's data is medication free. Therefore, whether his approach is applicable to other patients or not, additional analyses are required.

\section{References}

1. American Diabetes Association (2020) Diabetes Care. 2020; 43 (suppl 1): s1-s212.

2. Tadej Battelino, Thomas Danne, Moshe Phillip (2019) 2-LB: CGM-Based Clinical Targets: Recommendations from the International Consensus on Time-in-Range (TIR). Diabetes. 2019 Jun; 68 (Supplement 1).

3. Vigersky R, McMahon C (2019) Diabetes Technol Ther 21(2): 81-85.

4. Beck RW, Bergenstal RM, Cheng P, et al. (2019) J Diabetes Sci Technol 13(4): 614-626.

5. Battelino T, Danne T, Bergenstal RM, et al. (2019) Diabetes Care 42(8): 1593-1603.

6. Hsu, Gerald C (2020) The influences of medication on diabetes control using TIR analysis (GH-Method: Mathphysical medicine).

7. Hsu, Gerald C (2020) Case study of a type 2 diabetes patient's time-in-range (15-min) analysis using GHMethod: Math-physical medicine.

8. Hsu, Gerald C. (2020); The analysis of the ADA defined TIR, TAR, and TBR based on 5-minute measurement intervals of the CGM sensor glucose data using GHMethod: Math-physical medicine.
Copyright: (C)2020 Gerald C. Hsu. This is an open-access article distributed under the terms of the Creative Commons Attribution License, which permits unrestricted use, distribution, and reproduction in any medium, provided the original author and source are credited. 